\title{
Methyl jasmonate-elicited herbivore resistance: does MeJA function as a signal without being hydrolyzed to JA?
}

\author{
Jinsong Wu $\cdot$ Lei Wang $\cdot$ Ian T. Baldwin
}

Received: 13 November 2007 / Accepted: 7 January 2008 / Published online: 23 January 2008

(C) Springer-Verlag 2008

\begin{abstract}
Treatment with methyl jasmonate (MeJA) elicits herbivore resistance in many plant species and over-expression of JA carboxyl methyltransferase (JMT) constitutively increases JA-induced responses in Arabidopsis. When wildtype (WT) Nicotiana attenuata plants are treated with MeJA, a rapid transient endogenous JA burst is elicited, which in turn increases levels of nicotine and trypsin proteinase inhibitors (TPIs) and resistance to larvae of the specialist herbivore, Manduca sexta. All of these responses are impaired in plants silenced in lipoxygenase 3 expression (asLOX3) but are restored to WT levels by MeJA treatment. Whether these MeJA-induced responses are directly elicited by MeJA or by its cleavage product, JA, is unknown. Using virus-induced gene silencing (VIGS), we silenced MeJAesterase (NaMJE) expression and found this gene responsible for most of the MeJA-cleaving activity in $N$. attenuata protein extracts. Silencing $N a M J E$ in as $L O X 3$, but not in WT plants, significantly reduced MeJA-induced nicotine levels and resistance to M. sexta, but not TPI levels. MeJAinduced transcript levels of threonine deaminase (NaTD) and phenylalanine ammonia lyase $(\mathrm{NaPAL1})$ were also decreased in VIGS MJE (asLOX3) plants. Finally the performance of $M$. sexta larvae that fed on plants treated with JA or MeJA demonstrated that silencing NaMJE inhibited MeJA-induced but not JA-induced resistance in as $L O X 3$ plants. From these results, we conclude that the resistance
\end{abstract}

Electronic supplementary material The online version of this article (doi:10.1007/s00425-008-0690-8) contains supplementary material, which is available to authorized users.

J. Wu $\cdot$ L. Wang $\cdot$ I. T. Baldwin $(\bowtie)$

Department of Molecular Ecology,

Max Planck Institute for Chemical Ecology,

Hans-Knöll-Strasse 8, 07745 Jena, Germany

e-mail: Baldwin@ice.mpg.de elicited by MeJA treatment is directly elicited not by MeJA but by its de-methylated product, JA.

Keywords MeJA esterase (NaMJE) - Methyl jasmonate (MeJA) - Jasmonate (JA) - Nicotiana attenuata .

Manduca sexta

\section{Introduction}

Methyl jasmonate (MeJA), jasmonic acid (JA) and its amino acid conjugates, collectively referred to as jasmonates, are important cellular regulators mediating diverse developmental processes including root growth, pollen production, and plant resistance to insects and pathogens (Creelman and Mullet 1997; Kessler and Baldwin 2002). Jasmonates are synthesized in plants via the octadecanoid pathway (Creelman and Mullet 1997). Briefly, linolenic acid is oxygenated by lipoxygenase (LOX) and then converted to 12-oxo-phytodienoic acid (12-oxo-PDA) by allene oxide synthase (AOS) and allene oxide cyclase (AOC). JA is synthesized from 12-oxo-PDA through reduction and three steps of $\beta$-oxidation, and then catabolized further to form its volatile counterpart, MeJA (Seo et al. 2001), and numerous conjugates including JA-isoleucine (JA-Ile, Staswick and Tiryaki 2004; Kang et al. 2006; Wang et al. 2007a, b). MeJA is one of the JA metabolites proposed to play an important role in inter- and intra-plant signaling (Farmer and Ryan 1990; Seo et al. 2001; Karban et al. 2000; Kessler et al. 2006; Baldwin et al. 2006). When plants are exposed to volatile MeJA, they quickly elicit a series of JA mediated defense responses and MeJA treatment is the most commonly used means of eliciting herbivore resistance in many different plant species (McConn et al. 1997; Baldwin 1998; $\mathrm{Li}$ et al. 2002). However, it is 
still not known how plants elicit herbivore resistance traits in response to MeJA exposure.

When Nicotiana attenuata is attacked by herbivores, it produces both volatiles to recruit the herbivores' natural enemies (Kessler and Baldwin 2001; Mattiacci et al. 1995) and secondary metabolites that function as direct defenses, such as the neurotoxin nicotine (Baldwin 1999; Steppuhn et al. 2004), and trypsin proteinase inhibitors (TPIs, Zavala et al. 2004). JA signaling plays a central role in these responses. Silencing a key gene involved in supplying fatty acid hydroperoxides for JA biosynthesis, lipoxygenase 3 (NaLOX3), reduces the wound- and herbivore-induced accumulation of JA, but not the constitutive levels of JA. This JA deficiency inhibits the elicitation of direct (TPIs and nicotine) and indirect (volatiles) defenses and reduces $N$. attenuata's resistance to attack by larvae of the specialist herbivore of $N$. attenuata, Manduca sexta (Halitschke and Baldwin 2003), and makes the plants susceptible to two new herbivores, the leaf-chewing beetle Diabrotica undecimpunctata and the piercing-sucking leafhopper Empoasca spp. (Kessler et al. 2004). Interestingly, when NaLOX3silenced plants are treated with MeJA, their ability to produce nicotine and herbivore-resistance traits is fully restored (Halitschke and Baldwin 2003), suggesting that the exogenous MeJA treatment is sufficient to elicit most JA responses. However, these results raise an important question: does the exogenously supplied MeJA function directly as a signal or must it first be hydrolyzed to JA? Both JA and MeJA are elicitors of defense responses when applied exogenously, and they induce almost the same set of genes (Taki et al. 2005), but the nature of the endogenous signal remains unclear as both are rapidly interchangeable. Overexpression of JA carboxyl methyltransferase (JMT) in Arabidopsis increases endogenous MeJA levels 3-fold without altering JA levels and results in the constitutive expression of JA-responsive genes, including VSP and PDF1.2 (Seo et al. 2001). These results suggest that MeJA rather than JA elicits systemically transmitted defense responses. Similarly, a long-standing debate about whether salicylic acid (SA) or its methyl ester, MeSA, was the elicitor of systemically acquired resistance to pathogen attack was recently resolved by the ingenious idea of grafting together combinations of plants altered in their expression of either the MeSA-esterase and SA-methyl transferases. MeSA was confirmed to be the critical mobile signal for SAR (Park et al. 2007).

The discovery of MeJA-esterase (MJE, Stuhlfelder et al. 2002, 2004), which hydrolyzes MeJA to JA, provides a means of determining whether MeJA is the elicitor of MeJA-elicited herbivore resistance. Since MeJA and JA treatment of plants is known to elicit endogenous JA production (Ziegler et al. 2001; Miersch and Wasternack 2000; Pluskota et al. 2007), the hypothesis is best tested in plants reduced in their endogenous JA production. We tested the hypothesis that MeJA-elicited herbivore resistance is actually elicited after de-esterification to JA. We used virusinduced gene silencing (VIGS) to silence MeJA esterase (NaMJE) transcripts in wild-type (WT) and asLOX3 $N$. attenuata plants (Halitschke and Baldwin 2003) with a tobacco rattle virus-based system that had been optimized for N. attenuata (Saedler and Baldwin 2004) and measured defense responses, transcripts and herbivore performance in plants elicited with MeJA or JA treatments.

\section{Materials and methods}

\section{Plant growth}

We used seeds of the 21st generation of an inbred line of $N$. attenuata Torr. Ex Watts (synonymous with Nicotiana torreyana: Solanaceae) for transformation. Seed germination and plant growth were conducted as described by Krügel et al. (2002). In brief, seeds were sterilized and germinated on agar with Gamborg B5 (Duchefa http://www.duchefa.com) after soaking in a 1:50 (v/v) diluted liquid smoke (House of Herbs, Passaic, NY, USA) and $1 \mathrm{mM}$ of gibberellic acid $\left(\mathrm{GA}_{3}\right)$. After 10 days, seedlings were planted into soil in Teku pots. Once established, plants were transferred to $1 \mathrm{~L}$ pots in soil and grown in a growth chamber at $22^{\circ} \mathrm{C}$, under $16 \mathrm{~h}$ of light supplemented with Philips Sun-T Agro $400 \mathrm{Na}$ lights (Philips, http://www.philips.com) or not.

\section{Isolating NaMJE}

A 312 bp fragment of the NaMJE cDNA sequence was cloned with primers (MJE30_for: 5'-GCTAGTTCATGG AGCTTGTC-3' and MJE341_rev: 5'-TTAGGACCAGG CATGAAAGC-3'), the design of which was based on the sequence similarity of EST (DB679695) and LeMJE (AY455313). After the first round of PCR with AP primer (5'-GCCACGCGTCGACTAGTACTTTTTTTTT TTTTTTTT-3') and primer MJE30_for, 3 terminal cDNA was amplified with primer MJE 49_for (5'-CACGGTGCA TGGTGTTGGTA-3') and primer NAP (5'-GCCACGCGT CGACTAGTAC- $\left.3^{\prime}\right)$. The 5 terminal cDNA was obtained with primer MJE0_for (5'-AGATGACATGGAAAAGG GT-3') and primer MJE341_rev. All cDNA fragments were cloned into a pGEM-T EASY vector (Promega, http:// www.promega.com) and sequenced.

\section{Generation of VIGS plants}

A 312 bp fragment of the NaMJE cDNA sequence, which was amplified by primers MJE30_for and MJE341_rev, was cloned into the pTV00. The pTV00 vector is a $5.5-\mathrm{kb}$ plas- 
mid with an origin of replication for Escherichia coli and A. tumefaciens and a gene for kanamycin resistance (Ratcliff et al. 2001). The A. tumefaciens (strain GV3101)-mediated transformation procedure was described previously (Saedler and Baldwin 2004). To monitor the progress of VIGS, we silenced phytoene desaturase, a gene that oxidizes and cyclizes phytoene to $\alpha$ - and $\beta$-carotene. These compounds are subsequently converted into the xanthophylls of the antenna pigments of the photosystems of plants, resulting in the visible bleaching of green tissues (Saedler and Baldwin 2004). When the leaves of phytoene desaturase-silenced plants began to bleach ( 5 weeks after germination), leaves of NaMJE-silenced (VIGS MJE) and empty vector-inoculated (EV) plants from both WT and asLOX3 plants were used.

\section{Caterpillar performance}

Eggs of M. sexta were acquired from North Carolina State University (http://www.ncsu.edu) and kept in a growth chamber (Snijders Scientific, http://www.snijders-tilburg.nl) at $26^{\circ} \mathrm{C} 16 \mathrm{~h}$ light, $24^{\circ} \mathrm{C} 8 \mathrm{~h}$ darkness, until the larvae hatched. Freshly hatched neonates were placed directly on the source-sink transition leaves 3 days after MeJA treatment. In each treatment, 20 larvae were weighed after the indicated times.

Analysis of nicotine levels and TPI activity

Nicotine was extracted and quantified by HPLC as described in (Keinanen et al. 2001). Trypsin proteinase (TPI) activity was analyzed by radial diffusion activity as described in (van Dam et al. 2001).

\section{JA and JA-Ile measurements}

JA and JA-Ile were extracted and quantified by LC/MS as described in (Wang et al. 2007a). In brief, about $200 \mathrm{mg}$ of leaf tissues from each sample was homogenized on a FastPrep homogenizer (http://www.thermo.com) with $1 \mathrm{~mL}$ of ethyl acetate spiked with $200 \mathrm{ng}$ of $1,2-{ }^{13} \mathrm{C}-\mathrm{JA}, \mathrm{D}_{4}$-SA and p-coumaric acid (PCA) in FastPrep tubes. After being centrifuged, the supernatants were transferred to fresh $2 \mathrm{~mL}$ tubes and evaporated on a vacuum concentrator. The residue was resuspended in $0.5 \mathrm{~mL}$ of $70 \%$ methanol (v/v) and centrifuged at maximum speed for $5 \mathrm{~min}$. The supernatants were analyzed for JA, JA-Ile, and SA with a 1200L LC/ MS/MS system (Varian, http://www.varianinc.com).

\section{SYBR green real-time PCR assay (qPCR)}

Total RNA was extracted with TRI Reagent (Sigma, http:// www.sigmaaldrich.com) according to the manufacturer's instructions, and cDNA was prepared from $500 \mathrm{ng}$ total
RNA with multiScribeTM reverse transcriptase (Applied Biosystems, http://www.appliedbiosys.com). The primers for NaMJE mRNA expression detection by qPCR were as follows:

NaMJE forward primer:5'-GCTTTCATGCCTGGTCC TAA-3',

NaMJE reverse primer:5'-GACCTTTCTCCTGTCCG TTG-3',

NaTPI forward primer:5'-TCAGGAGATAGTAAATATG GCTGTTCA -3',

NaTPI reverse primer:5' -ATCTGCATGTTCCACATTGC TTA-3',

NaTD forward primer:5'-TAAGGCATTTGATGGGA GGC-3',

NaTD reverse primer:5'-TCTCCCTGTTCACGATAATG GAA-3',

NaPAL1 forward primer:5'-TTTGCATACGCTGATGA CGC-3',

NaPAL1 reverse primer:5'-TGGAAGATAGAGCTGTTC GCG-3',

NaActin forward primer:5'-GGTCGTACCACCGGTATT GTG-3',

NaActin reverse primer:5'-GTCAAGACGGAGAATGGC ATG-3'.

Real-time PCR was performed on an ABI PRISM 7700 Sequence Detection System (qPCR Core Kit, Eurogentec, http://www.eurogentec.com) with NaActin for normalization and according to the manufacturer's instructions under the following cycle conditions: $10 \mathrm{~min} 95^{\circ} \mathrm{C} ; 40$ cycles: $30 \mathrm{~s} 95^{\circ} \mathrm{C}, 30 \mathrm{~s} 55^{\circ} \mathrm{C}$.

MeJA esterase activity assay

Around $200 \mathrm{mg}$ leaf sample was ground in liquid nitrogen, and total proteins were extracted with buffer $(100 \mathrm{mM}$ Tris, $5 \%$ polyvinyl polypyrrolidone, $0.2 \%$ phenylthio urea, and $0.5 \%$ diethyl dithio carbamate, $\mathrm{pH}$ 7.6). A standard enzyme incubation mixture (total volume $50 \mu \mathrm{L}$ ) contained $10 \mu \mathrm{L}$ total protein $(2 \mu \mathrm{g}), 2 \mu \mathrm{L}$ MeJA $(2.2 \mu \mathrm{g}$ in $10 \%$ ethanol), and $38 \mu \mathrm{L} 100 \mathrm{mM}$ Tris (pH 7.6). Reactions were performed at $40^{\circ} \mathrm{C}$ as suggested as the optimum temperature by Stuhlfelder et al. (2002) for 0, 20, and $60 \mathrm{~min}$ and terminated by chilling on ice. One milliliter of ethyl acetate (spiked with internal JA standard ${ }^{13} \mathrm{C}_{2}$-JA $200 \mathrm{ng}$ ) was added quickly. The product JA from cleaved MeJA was quantified by LC/MS/MS as described in (Wang et al. 2007a). From the concentration of JA produced, we obtained the amount of cleaved MeJA. We also performed reactions for negative controls with only $10 \mu \mathrm{L}$ total proteins of EV and VIGS MJE plants individually or only MeJA in Tris buffer at the same condition for $60 \mathrm{~min}$, the results showed that the amount of JA produced by these reactions is under the detection limit of our LC/MS/MS. 


\section{Results}

Silencing NaMJE dramatically reduces the hydrolysis of MeJA in protein extracts

The methyl jasmonate esterase (NaMJE, EU196055) cDNA was cloned by RT-PCR using primers designed from $M J E$ in tomato ( $L e M J E)$. The predicted amino acid sequence of NaMJE shares $80 \%$ sequence identity with LeMJE (AY455313, Fig. S1). We used the VIGS system to silence NaMJE mRNA in WT and as $L O X 3$ plants. Plants were inoculated with Agrobacterium-harboring TRV constructs that contained an empty vector (EV) or a 312-bp NaMJE fragment (VIGS MJE). After 14 days, when the visible bleaching of green tissues had been observed for 3 days in plants inoculated with phytoene desaturase (PDS), a gene required for the biosynthesis of xanthophylls of the antenna pigments, NaMJE transcripts were analyzed with qRT-PCR in four replicate source-sink transition leaves of EV and VIGS MJE plants and normalized to levels of NaActin. The results show that VIGS $M J E$ plants had only $16 \%$ of the NaMJE transcripts that EV plants had in both WT and as $L O X 3$ backgrounds (Fig. 1a).

To determine if the NaMJE we silenced was responsible for hydrolyzing MeJA to free JA, we measured the MeJAcleaving activity of proteins extracted from leaves of EV and VIGS MJE plants (Fig. 1b). In proteins extracted from EV as $L O X 3$ plants, $45 \mu \mathrm{g}$ MeJA was cleaved to free JA by $1 \mathrm{mg}$ EV proteins after $20 \mathrm{~min}$ under standard conditions, while only $9 \mu \mathrm{g}$ MeJA was cleaved by $1 \mathrm{mg}$ of proteins extracted from VIGS MJE asLOX3 plants (unpaired $t$ test, $P=0.0002)$; after $60 \mathrm{~min}$, the MeJA cleaved by VIGS $M J E$ was only $16 \%$ of the amount that EV proteins had cleaved (unpaired $t$ test, $P=0.0003$ ). Dramatically reduced MeJAcleaving activity was also found in VIGS MJE proteins in WT background (Fig. 1b). These results demonstrate that NaMJE is largely responsible for the MeJA-cleaving activity in N. attenuata leaves.

Silencing NaMJE inhibits MeJA-induced resistance to $M$. sexta in as $L O X 3$ plants but not in WT plants

To determine if silencing NaMJE influenced MeJAinduced resistance to $M$. sexta larvae, the source-sink transition leaves of EV and VIGS MJE plants were treated with lanolin paste (lanolin) or $75 \mu \mathrm{g}$ MeJA in lanolin (MeJA). After 4 days, $M$. sexta larvae were placed on these leaves and allowed to feed for 12 days. As expected, larvae feeding on lanolin-treated as $L O X 3$ plants gained significantly more mass than those on lanolin-treated WT plants and MeJA treatment dramatically reduced the $M$. sexta larval mass on EV plants: larvae that fed on MeJA-treated plants gained only 60.5 and $54 \%$ of the mass of those that fed on
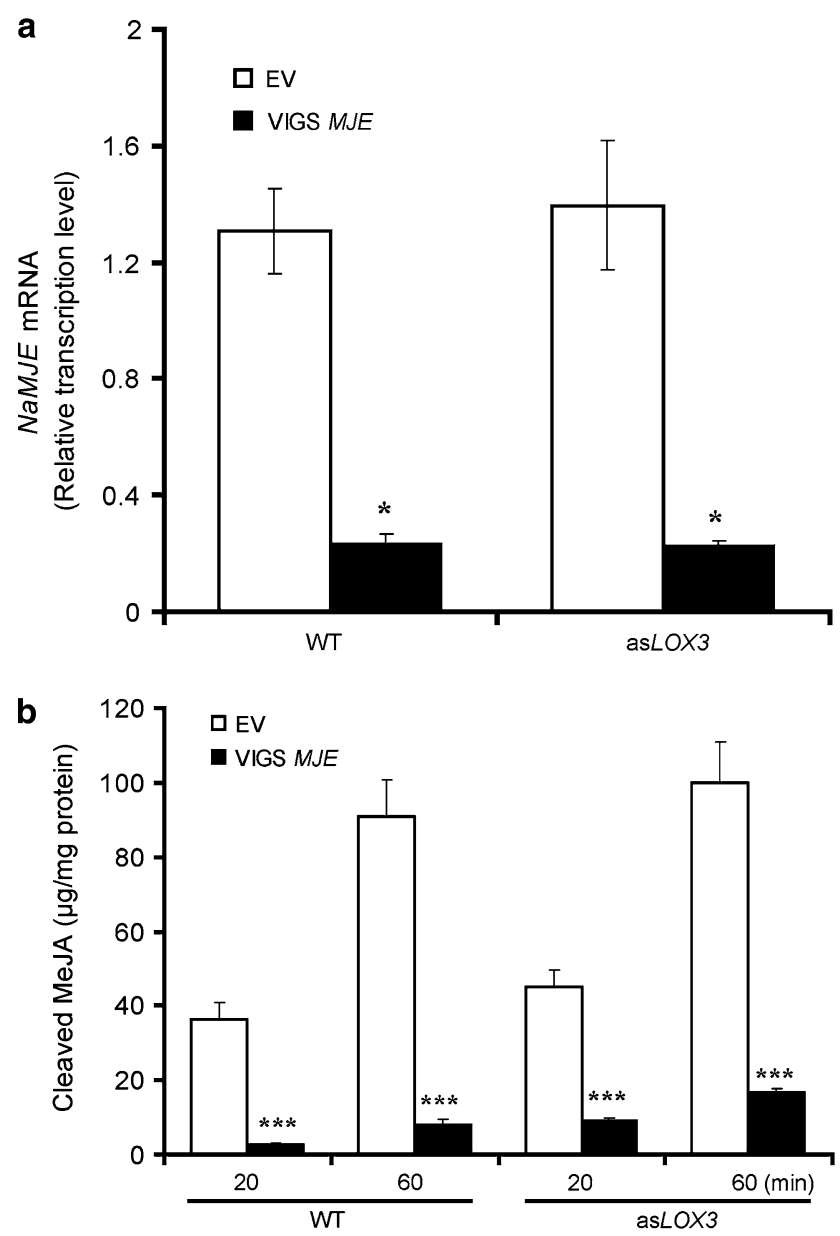

Fig. 1 Silencing NaMJE reduces the MeJA-cleaving activity. WT and as $L O X 3$ plants were inoculated with Agrobacterium-harboring TRV constructs, which contained an empty vector (EV) or a 312-bp NaMJE fragment (VIGS MJE). After 14 days, four replicate source-sink transition leaves were harvested. a: Mean $( \pm \mathrm{SE})$ NaMJE transcript accumulation in source-sink transition leaves of EV and VIGS MJE plants from both WT and asLOX3 backgrounds as analyzed with qRT-PCR normalized to levels of NaActin. b: Mean $( \pm \mathrm{SE})$ amount of MeJA hydrolyzed to free JA in four replicate total crude protein samples prepared from four individual EV and VIGS MJE-innoculated plants (WT and as $L O X 3$ background) after incubation with MeJA for 20 and $60 \mathrm{~min}$. Asterisks indicate the level of significant difference between EV and VIGS MJE plants (unpaired $t$ test: $* P<0.05$; ** $P<0.001$; *** $P<0.005)$

lanolin-treated WT and as $L O X 3$ plants, respectively (Fig. 2). Larvae performed equally well on EV and VIGS $M J E$ plants treated with lanolin in either WT or as $L O X 3$ genetic backgrounds (Fig. 2). However, silencing NaMJE completely inhibited MeJA-induced resistance in as $L O X 3$ plants: larvae that fed on MeJA-treated VIGS MJE plants gained the same mass as those that fed on plants treated with lanolin (Fig. 2), but gained 50\% more mass than those that fed on EV plants treated with MeJA (Fig. 2; unpaired $t$ test, $P=0.04)$. These results demonstrate that in asLOX3 plants, silencing NaMJE is sufficient to block MeJAinduced resistance. 


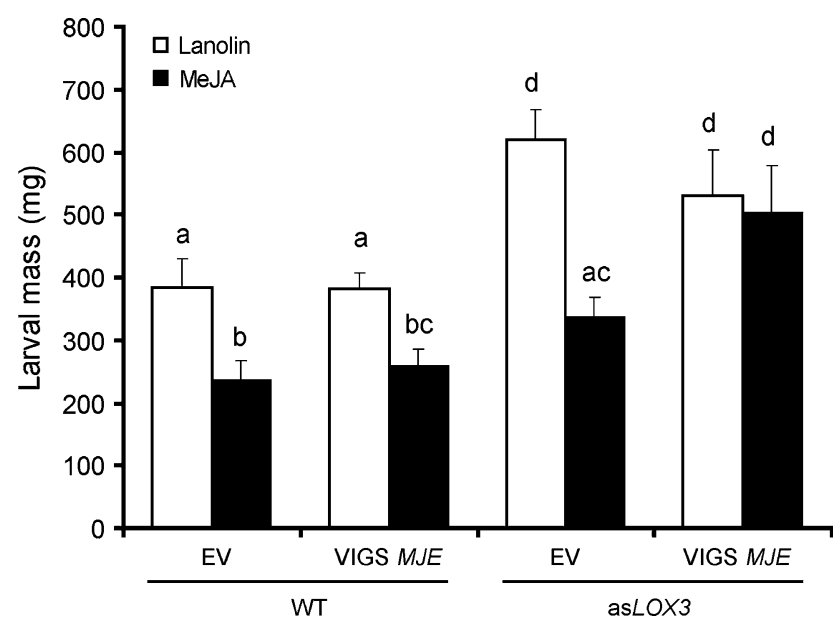

Fig. 2 Silencing NaMJE inhibits MeJA-induced resistance to $M$. sexta in as $L O X 3$ plants but not WT plants. Mean $( \pm \mathrm{SE})$ mass of 18 replicate $M$. sexta larvae after 12 days of feeding individually on EV and VIGS $M J E \mathrm{WT}$ and as $L O X 3$ plants. The source-sink transition leaves of EV and VIGS MJE plants were treated with lanolin or $75 \mu \mathrm{g}$ MeJA in lanolin. After 4 days, M. sexta larvae were placed on these leaves. Different letters indicate statistically significant differences between treatments (Fisher's PLSD test; $P<0.05$ )

Silencing NaMJE impairs MeJA-induced nicotine, NaPAL1 and $T D$ transcripts but not TPI responses in as $L O X 3$ plants

To understand why silencing NaMJE transcripts had such a strong effect on caterpillar performance in MeJA-induced asLOX3 but not WT plants, we measured MeJA-induced nicotine and TPI levels. After being treated with MeJA for 3 days, all EV plants had significantly increased nicotine levels (Fig. 3a). In WT plants, VIGS MJE plants accumulated the same amount of nicotine as EV plants had after MeJA treatment. However, nicotine levels of MeJA-treated VIGS MJE asLOX3 plants were only $66 \%$ of those in MeJA-treated EV plants (Fig. 3a; unpaired $t$ test, $P=0.01$ ).

MeJA treatment elicited the same amount of TPI activity in EV and VIGS MJE plants in both WT and asLOX3 backgrounds (Fig. 3b), suggesting that silencing NaMJE had no effect on the elicitation of TPIs by MeJA. Measurements of TPI transcripts in as $L O X 3$ plants $8 \mathrm{~h}$ after MeJA treatment also revealed no significant differences between $\mathrm{EV}$ and VIGS MJE plants (unpaired $t$ test, $P=0.5$ ).

To better understand why silencing NaMJE in as $L O X 3$ plants had such a large effect on MeJA-induced herbivore resistance, we compared transcript levels of phenylalanine ammonia lyase (PAL1), a well-known gene marker of phenolic-based defense responses, in the source-sink transition leaves of EV and VIGS MJE plants after MeJA treatment. $P A L 1$ was dramatically induced in EV plants. After $4 \mathrm{~h}$ of MeJA treatment, levels of PAL1 transcripts in VIGS MJE plants were only $21 \%$ of those in EV plants (Fig. 4a;
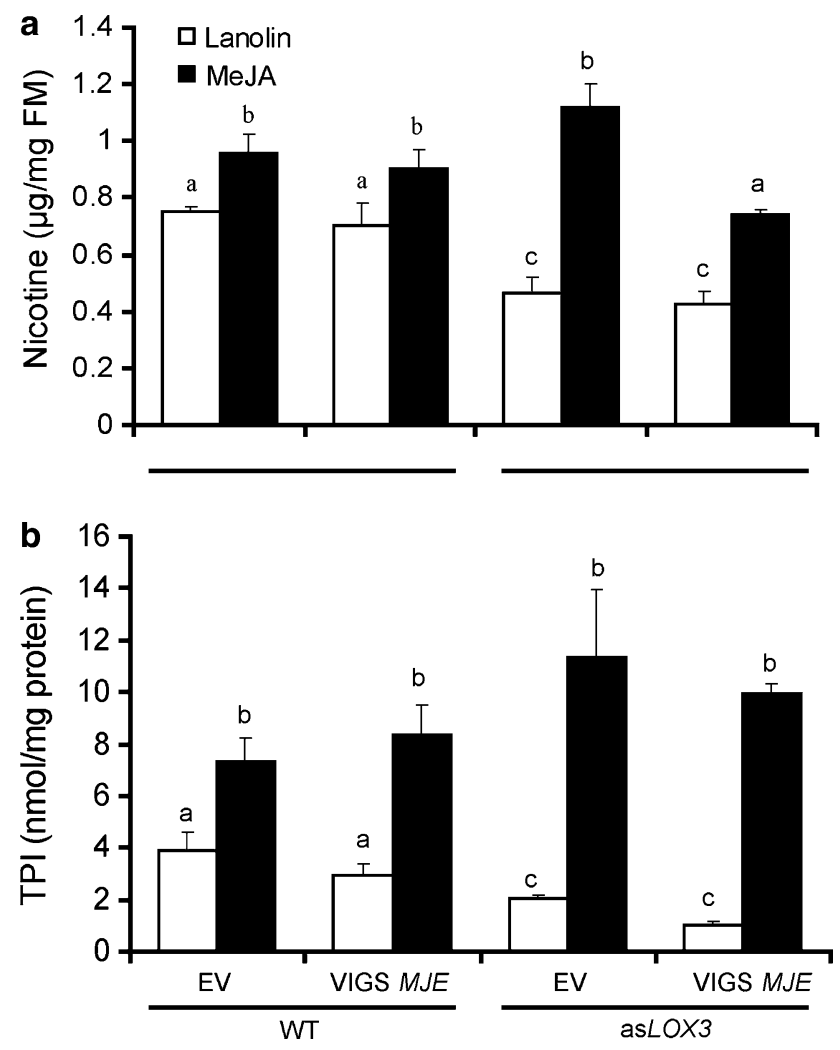

Fig. 3 Effects of silencing NaMJE on MeJA-induced levels of nicotine and TPI in WT and as $L O X 3$ plants. The source-sink transition leaves of EV and VIGS MJE plants were treated with lanolin or $75 \mu \mathrm{g}$ MeJA in lanolin. After 3 days, four replicate leaf samples per treatment were harvested and analyzed for: a Mean $( \pm \mathrm{SE})$ nicotine levels and $\mathbf{b}$ mean $( \pm$ SE) TPI levels. Different letters indicate statistically significant differences among treatments (Fisher's PLSD test; $P<0.05$ )

unpaired $t$ test, $P=0.005)$; after $8 \mathrm{~h}$, levels were $25 \%$ of those in EV plants (Fig. $4 \mathrm{a}$; unpaired $t$ test, $P=0.05$ ).

Threonine deaminase $(T D)$ catalyzes the conversion of Thr to a-keto butyrate in Ile biosynthesis, and is strongly elicited in MeJA-elicited leaves in N. attenuata where it supplies the Ile required for JA-Ile production (Kang et al. 2006). MeJA treatments dramatically increased NaTD transcripts in EV plants; however, in VIGS MJE plants, NaTD transcript levels were only $9 \%$ of those in EV plants $8 \mathrm{~h}$ after MeJA treatment (Fig. 4b; unpaired $t$ test, $P=0.03$ ).

Silencing NaMJE inhibits MeJA-induced but not JAinduced resistance to $M$. sexta in as $L O X 3$ plants

If it is true that MeJA functions as a signal prior to being hydrolyzed to JA, silencing NaMJE should inhibit MeJAinduced resistance but have no effect on JA-induced resistance. Due to its polarity, JA does not elicit resistance when added to plants in a lipophilic lanolin paste. Therefore, aqueous solutions of JA and MeJA were added to standardized puncture wounds in leaves of EV and VIGS MJE asLOX3 

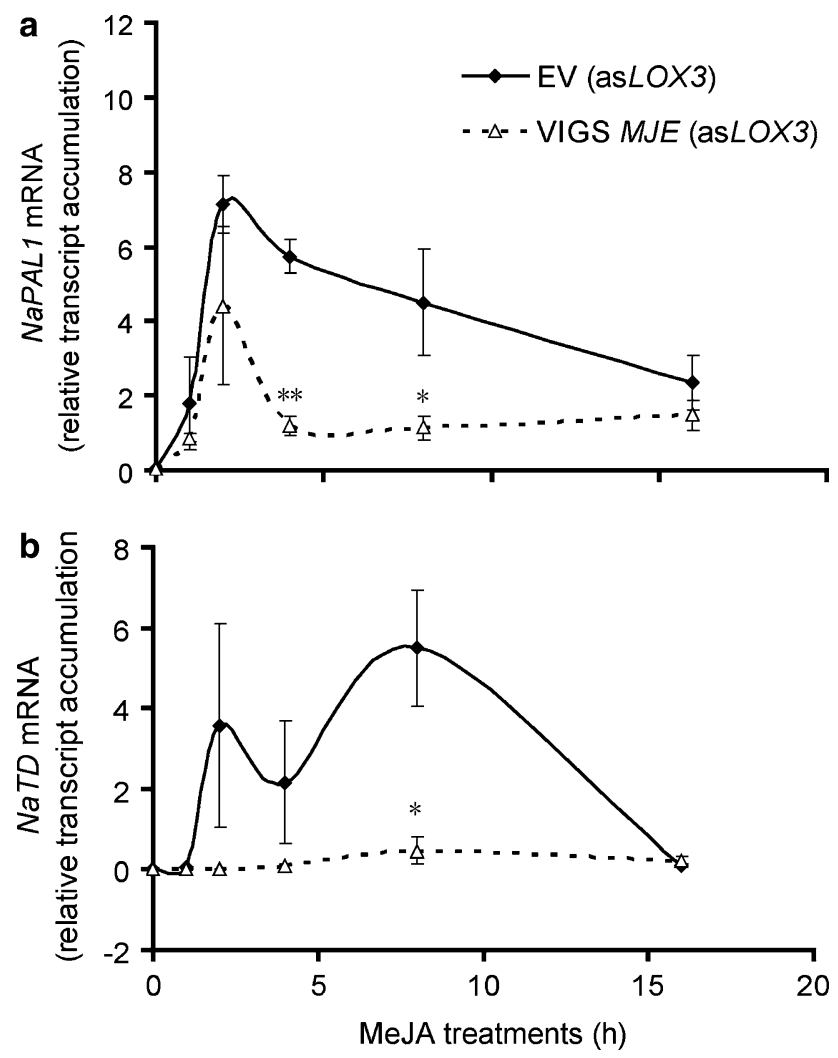

Fig. 4 Silencing NaMJE inhibited MeJA-induced NaPAL1 and TD transcripts in as $L O X 3$ plants. Four replicate source-sink transition leaves of EV and VIGS MJE plants were treated with $75 \mu \mathrm{g}$ MeJA in lanolin and harvested after $0,1,2,4,8$, and $16 \mathrm{~h}$. Mean $( \pm \mathrm{SE})$ a NaPAL1 and b TD transcription levels were analyzed with qRT-PCR normalized to levels of NaActin. Asterisks indicate the level of significant difference between EV and VIGS MJE plants, both treated with MeJA (unpaired $t$ test: $* P<0.05 ; * * P<0.005$ )

plants and we tested this hypothesis by measuring the performance of larvae. As expected, the mass of caterpillars that fed on EV plants treated with both JA and MeJA was dramatically reduced compared to the mass of those that fed on EV plants treated with water (Fig. 5). Larvae that fed on JA-treated VIGS MJE plants gained the same mass as those that fed on EV plants treated with JA; however, larvae gained significantly more mass when they fed on VIGS MJE plants than on EV plants treated with MeJA (Fig. 5).

\section{Discussion}

Staswick's pioneering work on MeJA-insensitive jarl mutant in Arabidopsis suggests that exogenously applied MeJA is first demethylated and then conjugated to Ile before it becomes an active inhibitor of root growth (Staswick et al. 1992; Staswick and Tiryaki 2004). Indeed, MeJA hydrolyzing enzyme activity occurs in all the plant species that have been examined to date (Stuhlfelder et al.

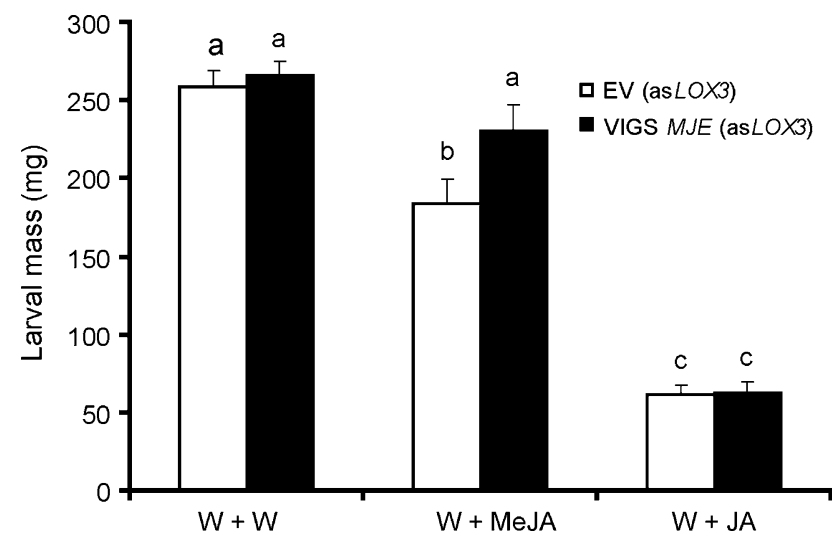

Fig. 5 Silencing NaMJE inhibited MeJA-induced but not JA-induced resistance to $M$. sexta in as $L O X 3$ plants The source-sink transition leaves of EV and VIGS MJE plants were wounded with a fabric pattern wheel; water (12.5\% ethanol), or $0.25 \mu \mathrm{mol} \mathrm{JA}$, or $0.25 \mu \mathrm{mol}$ MeJA were immediately applied to the puncture wounds. After 2 days, $18 \mathrm{M}$. sexta larvae were placed individually on the source-sink transition leaves and weighed 15 days after the start of feeding. Different letters indicate statistically significant differences between treatments (Fisher's PLSD test; $P<0.05$ )

2002). It is also reported that MeJA was rapidly hydrolyzed to JA and further metabolized like JA when tobacco BY-2 cells were treated with MeJA (Swiatek et al. 2004). In $N$. attenuata, a model plant with extensively studied herbivore-induced responses, we also detected high levels of MeJA-cleaving activity (Fig. 1b). Using the sequence similarity with tomato LeMJE (Stuhlfelder et al. 2004), we cloned NaMJE, a MeJA esterase gene that shared very high sequence similarity with LeMJE (Fig. S1). By silencing the expression of this gene, we demonstrated that NaMJE is largely responsible for the MeJA-cleaving activity of N. attenuata leaves (Fig. 1b).

MeJA treatment is the most commonly used means of eliciting herbivore resistance in many different plant species (McConn et al. 1997; Baldwin 1998; Li et al. 2002). However, it is still not known how herbivore resistance traits are elicited by MeJA treatment. We determined whether MeJA functions as a signal prior to being hydrolyzed to JA by investigating MeJA-induced defense responses in both WT and JA-deficient asLOX3 plants (Figs. S2, S3) with reduced MeJA-cleaving activity. Silencing NaMJE transcripts was sufficient to block most MeJA-induced responses in as $L O X 3$ but not WT plants, including the production of nicotine (Fig. 3), transcripts of NaPALI and NaTD (Fig. 4). Importantly, the dramatic decreases in mass that are usually observed in larvae that feed on MeJA-treated EV plants disappeared when NaMJE transcripts were silenced (Fig. 2). Furthermore, silencing NaMJE inhibited MeJA-induced but not JA-induced resistance in as $L O X 3$ plants (Fig. 5). Why is silencing NaMJE sufficient to block most MeJA-induced responses in as $L O X$ plants but not in WT plants? MeJA 
treatment of plants is known to elicit endogenous JA production (Ziegler et al. 2001; Miersch and Wasternack 2000). Without any treatments, asLOX3 plants have the same level of endogenous JA and JA-Ile as WT plants (Figs. S2a, S3). However, after MeJA treatments, asLOX3 plants accumulate lower levels of JA than do WT plants (Fig. S2b). These results are consistent with the reports in Halitschke and Baldwin (2003) that asLOX3 plants have the same basal levels of JA as WT plants do but accumulate half of the JA levels of WT plants after wounding, suggesting that the JA burst arises in part from de novo biosynthesis and in part from JA that is released from some unknown storage pool. Since NaMJE mRNA levels and MeJA cleaving activity are the same in EV (WT) and EV (asLOX3) plants, the higher levels of JA (Fig. S2b) elicited by MeJA in EV (WT) plants compared to EV (asLOX3) plants are likely due to the larger JA storage pool in WT plants. In light of these considerations, we conclude that most exogenous MeJA-induced herbivore responses are actually elicited after MeJA is demethylated to JA.

Trypsin proteinase inhibitors (TPIs) play a role as a direct defensive against herbivores in $N$. attenuata (Zavala et al. 2004). The activities and transcripts of TPIs can be dramatically induced by MeJA treatments. Preston et al. (2004) showed that the application of $5 \mu \mathrm{g}$ MeJA was sufficient to elicit a significant increase in TPI activity, which suggested that TPIs are very sensitive to MeJA treatment, which may explain why even a reduction of $84 \%$ of the MeJA-cleaving activity was not sufficient to inhibit the MeJA-induced TPI response in as $L O X 3$ plants. Therefore from these results, we cannot determine whether the accumulations of TPIs were elicited by MeJA directly or after demethylation to JA.

Silencing NaMJE completely blocked the MeJA-induced resistance to $M$. sexta larvae in as $L O X 3$ plants (Fig. 3). Although the levels of MeJA-induced nicotine were significantly lower in VIGS MJE plants than in EV plants, the reduction in the nicotine response was not likely sufficiently large to account for the increase in herbivore performance (Steppuhn et al. 2004). Two additional possible explanations are suggested by the results: (1) the reduction in NaPALI transcripts could reflect reductions in an unknown phenolicbased defense; (2) reduced NaTD transcripts may reflect decreased TD activity, which may function post-ingestively as an antinutritive defense that limits the supply of Thr needed for herbivore growth (Chen et al. 2005).

In summary, our results demonstrate that most herbivore-resistant responses are elicited by JA when plants were treated with exogenously applied MeJA.

Acknowledgments We thank Dr. K. Gase, Thomas Hahn, Susan Kutschbach, Antje Wissgott for sequencing NaMJE and making the VIGS constructs; Jianqiang Wu for help in the laboratory and discussion; Eva Rothe and Dr. M. Schöttner for help in the analysis of JA and JA-Ile; Emily Wheeler for improving the manuscript. We thank the funding from the Virtual Institute Biotic Interaction (ViBi) by the Helmholtz Association for JW's research stipend, and the Max Planck Society for funding all other aspects of the research.

\section{References}

Baldwin IT (1998) Jasmonate-induced responses are costly but benefit plants under attack in native populations. Proc Natl Acad Sci USA 95:8113-8118

Baldwin IT (1999) Inducible nicotine production in native Nicotiana as an example of adaptive phenotypic plasticity. J Chem Ecol 25:3-30

Baldwin IT, Halitschke R, Paschold A, von Dahl CC, Preston CA (2006) Volatile signaling in plant-plant interactions: "talking trees" in the genomics era. Science 311:812-815

Chen H, Wilkerson CG, Kuchar JA, Phinney B S, Howe GA (2005) Jasmonate-inducible plant enzymes degrade essential amino acids in the herbivore midgut. Proc Natl Acad Sci USA 102:1923719242

Creelman RA, Mullet JE (1997) Biosynthesis and action of jasmonates in plants. Annu Rev Plant Physiol Plant Mol Biol 48:355-381

Farmer EE, Ryan CA (1990) Interplant communication: airborne methyl jasmonate induces synthesis of proteinase inhibitors in plant leaves. Proc Natl Acad Sci USA 87:7713-7716

Halitschke R, Baldwin IT (2003) Antisense LOX expression increases herbivore performance by decreasing defense responses and inhibiting growth-related transcriptional reorganization in Nicotiana attenuata. Plant J 36:794-807

Kang JH, Wang L, Giri A, Baldwin IT (2006) Silencing threonine deaminase and JAR4 in Nicotiana attenuata impairs jasmonic acid-isoleucine-mediated defenses against Manduca sexta. Plant Cell 18:3303-3320

Karban R, Baldwin IT, Baxter KJ, Laue G, Felton GW (2000) Communication between plants: induced resistance in wild tobacco plants following clipping of neighboring sagebrush. Oecologia 125:6671

Keinanen M, Oldham NJ, Baldwin IT (2001) Rapid HPLC screening of jasmonate-induced increases in tobacco alkaloids, phenolics, and diterpene glycosides in Nicotiana attenuata. J Agric Food Chem 49:3553-3558

Kessler A, Baldwin IT (2001) Defensive function of herbivore-induced plant volatile emissions in nature. Science 291:2141-2144

Kessler A, Baldwin IT (2002) Plant responses to insect herbivory: the emerging molecular analysis. Annu Rev Plant Biol 53:299-328

Kessler A, Halitschke R, Baldwin IT (2004) Silencing the jasmonate cascade: Induced plant defenses and insect populations. Science 305:665-668

Kessler A, Halitschke R, Diezel C, Baldwin IT (2006) Priming of plant defense responses in nature by airborne signaling between Artemisia tridentata and Nicotiana attenuata. Oecologia 148:280292

Krügel T, Lim M, Gase K, Halitschke R, Baldwin IT (2002) Agrobacterium-mediated transformation of Nicotiana attenuata, a model ecological expression system. Chemoecology 12:177-183

Li C, Williams MM, Loh YT, Lee GI, Howe GA (2002) Resistance of cultivated tomato to cell content-feeding herbivores is regulated by the octadecanoid-signaling pathway. Plant Physiol 130:494503

Mattiacci L, Dicke M, Posthumus MA (1995) beta-Glucosidase: an elicitor of herbivore-induced plant odor that attracts host-searching parasitic wasps. Proc Natl Acad Sci USA 92:2036-2040

McConn M, Creelman RA, Bell E, Mullet JE, Browse J (1997) Jasmonate is essential for insect defense in Arabidopsis. Proc Natl Acad Sci 94:5473-5477 
Miersch O, Wasternack C (2000) Octadecanoid and jasmonate signaling in tomato (Lycopersicon esculentum Mill.) leaves: endogenous jasmonates do not induce jasmonate biosynthesis. Biol Chem 381:715-722

Park SW, Kaimoyo E, Kumar D, Mosher S, Klessig DF (2007) Methyl salicylate is a critical mobile signal for plant systemic acquired resistance. Science 318:113-116

Pluskota W, Qu N, Maitrejean M, Boland W, Baldwin IT (2007) Jasmonates and mimics differentially elicit systemic defense responses in Nicotiana attenuata. J Exp Bot (in press)

Preston CA, Laue G, Baldwin IT (2004) Plant-plant signaling: application of trans- or cis-methyl jasmonate equivalent to sagebrush releases does not elicit direct defenses in native tobacco. J Chem Ecol 30:2193-2214

Ratcliff F, Martin-Hernandez AM, Baulcombe DC (2001) Technical Advance. Tobacco rattle virus as a vector for analysis of gene function by silencing. Plant $\mathrm{J}$ 25:237-245

Saedler R, Baldwin IT (2004) Virus-induced gene silencing of jasmonate-induced direct defences, nicotine and trypsin proteinaseinhibitors in Nicotiana attenuata. J Exp Bot 55:151-157

Seo HS, Song JT, Cheong JJ, Lee YH, Lee YW, Hwang I, Lee JS, Choi YD (2001) Jasmonic acid carboxyl methyltransferase: a key enzyme for jasmonate-regulated plant responses. Proc Natl Acad Sci USA 98:4788-4793

Staswick PE, Su W, Howell SH (1992) Methyl jasmonate inhibition of root growth and induction of a leaf protein are decreased in an Arabidopsis thaliana mutant. Proc Natl Acad Sci USA 89:68376840

Staswick PE, Tiryaki I (2004) The oxylipin signal jasmonic acid is activated by an enzyme that conjugates it to isoleucine in Arabidopsis. Plant Cell 16:2117-2127

Steppuhn A, Gase K, Krock B, Halitschke R, Baldwin IT (2004) Nicotine's defensive function in nature. PLoS Biol 2:E217
Stuhlfelder C, Lottspeich F, Mueller MJ (2002) Purification and partial amino acid sequences of an esterase from tomato. Phytochemistry 60:233-240

Stuhlfelder C, Mueller MJ, Warzecha H (2004) Cloning and expression of a tomato cDNA encoding a methyl jasmonate cleaving esterase. Eur J Biochem 271:2976-2983

Swiatek A, Van Dongen W, Esmans EL, Van Onckelen H (2004) Metabolic fate of jasmonates in tobacco bright yellow-2 cells. Plant Physiol 135:161-172

Taki N, Sasaki-sekimoto Y, Obayashi T, Kikuta A, Kobayashi K, Ainai T, Yagi K, Sakurai N, Suzuki H, Masuda T, Takamiya K, Shibata D, Kobayashi Y, Ohta H (2005) 12-Oxo-phytodienoic acid triggers expression of a distinct set of genes and play a role in wound-induced gene expression in Arabidopsis. Plant Physiol 139:1268-1283

van Dam NM, Horn M, Mares M, Baldwin IT (2001) Ontogeny constrains systemic protease inhibitor response in Nicotiana attenuata. J Chem Ecol 27:547-568

Wang L, Halitschke R, Kang JH, Berg A, Harnisch F, Baldwin IT (2007a) Independently silencing two JAR family members impairs levels of trypsin proteinase inhibitors but not nicotine. Planta 226:159-167

Wang L, Allmann S, Wu J, Baldwin IT (2007b) Comparisons of LOX3- and JAR4/6-silenced plants reveal that JA and JA-AA conjugates play different roles in herbivore resistance of Nicotiana attenuata. Plant Physiol (in press)

Zavala JA, Patankar AG, Gase K, Hui D, Baldwin IT (2004) Manipulation of endogenous trypsin proteinase inhibitor production in Nicotiana attenuata demonstrates their function as antiherbivore defenses. Plant Physiol 134:1181-1190

Ziegler J, Keinanen M, Baldwin IT (2001) Herbivore-induced allene oxide synthase transcripts and jasmonic acid in Nicotiana attenuata. Phytochemistry 58:729-738 